\title{
Subpicosecond coherent carrier-phonon dynamics in semiconductor heterostructures
}

\author{
T. Dekorsy, A. M. T. Kim, G. C. Cho, and H. Kurz \\ Institut für Halbleitertechnik II, Rheinisch Westfälische Technische Hochschule Aachen, Sommerfeldstraß 24, D-52074 Aachen, Germany \\ A. V. Kuznetsov* \\ Department of Physics, University of Florida, Gainesville, Florida 32611
}

A. Förster

Institut für Schicht-und Ionentechnik, Forschungszentrum Jülich, D-52425 Jülich, Germany

\begin{abstract}
Coherent longitudinal-optical phonons are generated in semiconductor heterostructures. The coupling of the coherent, longitudinal-optic (LO) phonons to collective carrier excitations oscillating parallel to the growth direction of $\mathrm{GaAs} / \mathrm{Al}_{0.36} \mathrm{Ga}_{0.64} \mathrm{As}$ quantum wells is investigated with femtosecond time-resolution. This coupling is found to be weak for small well widths and evolves towards the bulk plasmon phonon coupling at increased well widths. We present a theory for the dielectric function in the growth direction of the heterostructure and calculate the frequency response of the system on the pulsed optical excitation. It is shown that the observations are based on the coupling of coherent phonons to intersubband plasmons.
\end{abstract}

\section{INTRODUCTION}

Semiconductor quantum-well systems have been studied extensively with respect to the properties both of the twodimensional electron gas (2DEG) and the optical phonon system. ${ }^{1}$ The interaction between the $2 \mathrm{DEG}$ and the optical phonons determines physical properties relevant for electronic and optoelectronic devices such as energy relaxation and electronic transport in these systems. ${ }^{2}$ Femtosecond optical excitation allows the impulsive generation of coherent phonons, ${ }^{3-5}$ which are - in the case of III-V semiconductors - driven by the ultrafast separation of electron-hole pairs. ${ }^{3}$ By this technique, the interaction between LO phonons and the optically excited carriers can be investigated with subpicosecond time resolution. In bulk semiconductors, the coupling between coherent phonons and a dense carrier plasma has been studied in the time domain, opening the way to new insights into plasmon-phonon dynamics. ${ }^{5-7}$ Recently, coherent carrier oscillations in bulk GaAs with frequency far away from the LO phonon resonance have been observed. ${ }^{8}$ However, in two-dimensional heterostructures the excitation of coherent LO phonons has not been achieved up to now to our knowledge. ${ }^{9}$

Here we report on the interaction between optically excited carriers and coherently generated LO phonons in $\mathrm{GaAs} / \mathrm{Al}_{x} \mathrm{Ga}_{1-x} \mathrm{As}$ multiple quantum wells (MQW's) using femtosecond pump-probe spectroscopy. The polarization of the coherent LO phonons observed is directed parallel to the growth direction of the quantum wells due to a selective excitation mechanism based on the screening of a built-in field. Thus the plasmons observed via the coupling to the phonons are longitudinal collective excitations with the same direction of the dipole moment as the phonons, i.e., perpendicular to the plane of confinement. The energy levels of carriers in a quantum well are discrete in the growth direction. In contrast to plasmon oscillations in bulk, where the carriers can move continuously in $k$ space, the population of a single discrete level cannot give rise to an oscillating dipole moment directed parallel to the growth direction. Only if more than one eigenstate is populated can nonzero dipole matrix elements between the levels give rise to a timedependent dipole moment along the growth direction. These excitations have been denoted as intersubband plasmons. ${ }^{10}$

The coupled dynamics of coherent phonons and intersubband plasmons are traced by time resolving electro-optic index changes induced by the macroscopic coherent dipole oscillation in the sample. ${ }^{3,7}$ The coupling strength strongly depends on the width of the quantum wells and the population of the subbands of the wells. These observations are described by a theoretical model based on the ultrafast modification of the dielectric function by the population of several subbands of the quantum wells. The comparison of the experimental results with the theory indicates that the phonons are coupled to numerous intersubband plasmon modes of the quantum wells.

The first cw Raman experiments on plasmon-phonon coupling were reported by Mooradian and Wright in doped bulk GaAs. ${ }^{11}$ Time-resolved techniques involving above-band-gap excitation allow the simultaneous excitation of coherent phonons and carriers, while in cw techniques the carriers are usually introduced via doping. The dephasing of the coherent phonon amplitude is directly monitored in time domain, which allows an accurate determination of dephasing time constants down to the subpicosecond regime. For the $2 \mathrm{DEG}$ case, collective excitations have been studied intensively both experimentally and theoretically. ${ }^{12-16}$ In time domain, they have been observed recently in Raman scattering experiments. ${ }^{17}$ However, most of these works are concerned with $2 \mathrm{D}$ intrasubband plasmons with a wave vector $q_{\|}$, i.e., oscillating in the plane of electronic confinement. Since the carriers can move in a continuum in this plane, the plasmons can be calculated as a limit of 3D plasmons with a modified dispersion relation proportional to $q_{\|}^{1 / 2} \cdot{ }^{14}$ Collective excitations in quantum wells, which can be associated with a di- 
pole moment perpendicular to the layers, have been described as collective intersubband excitations. ${ }^{10,18-20}$ Evidence for this type of excitation has been found in Raman scattering experiments in modulation doped quantum wells. $^{13,20}$

As pointed out by Ryan and Tatham ${ }^{21}$ in a picosecond time-resolved study on 2D electron-phonon interaction, the very early stages of the interaction are not accessible in Raman experiments, since the increase of time resolution results in a decrease of spectral resolution. This is not the case for the type of measurements presented here.

\section{EXPERIMENTAL SETUP AND SAMPLES}

The experimental technique is reflective electro-optic sampling (REOS) with 40-50-fs time resolution. We use two laser sources. Laser pulses of 50-fs pulse duration at 2-eV photon energy are derived from a colliding pulse modelocked (CPM) dye laser. Pulses of 40-fs pulse duration at $1.5-\mathrm{eV}$ photon energy are obtained from a Kerr-lens modelocked Ti:sapphire laser. The experimental arrangement for both laser sources is the same. The laser pulses are split into an intense pump pulse and a weaker probe pulse, which is time delayed via a retroreflector mounted onto a speaker or a stepper delay stage. The linearly polarized probe beam has a polarization on the sample along one of the principal axes of the (100) GaAs surface. The reflected probe pulse is split

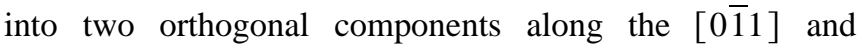
$[0 \overline{11}]$ crystal directions by a polarizing beam splitter. The two polarization components are detected with silicon $p-i-n$ diodes. The signals of each diode are fed into a difference amplifier (Tektronixs AM 502). Due to the symmetry of the GaAs electro-optic tensor, the difference signal directly gives the temporal changes of the electric field $\Delta E_{[100]}$ perpendicular to the sample surface via the linear electro-optic effect: $:^{3,22}$

$$
\frac{\Delta R_{e o}}{R_{0}}=\frac{4 n_{0}^{3}}{n_{0}^{2}-1} r_{41} \Delta E_{[100]},
$$

where $R_{0}$ is the unperturbed reflectivity, $n_{0}$ the unperturbed index of refraction, and $r_{41}$ the electro-optic coefficient of GaAs. The amplitude of the electro-optic reflectivity changes may be further influenced by the dispersion of $r_{41}$ for probing at different wavelengths. Coherent LO phonons with a dipole moment directed along the [100] direction are detected via their contribution to the linear electro-optic effect based on the associated macroscopic electric field changes and the atomic displacement. ${ }^{7}$ For a highly sensitive measurement of the phonon dynamics only, we apply a modified lock-in technique, where the time derivatives of the REOS signals are recorded. ${ }^{23}$ Here, the time delay is achieved via a stepper delay stage, while the speaker is driven at high frequencies $(\sim 600 \mathrm{~Hz})$ and modulates the pump-probe delay about 10 fs. All measurements are performed at room temperature.

For the excitation of coherent phonons, we investigate GaAs MQW's placed within the surface depletion region at the bare surface. The MQW's consist of 25 GaAs wells separated by $3-\mathrm{nm} \mathrm{Al}_{x} \mathrm{Ga}_{1-x} \mathrm{As}(x=0.36)$ barriers. The well width is varied from $15 \mathrm{~nm}$ to $30 \mathrm{~nm}$ in steps of $5 \mathrm{~nm}$. The samples are slightly background $\mathrm{p}$ doped, which results in a

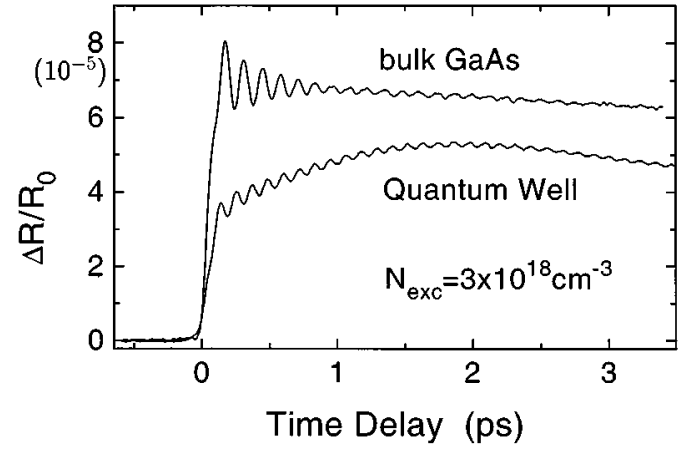

FIG. 1. REOS signals from a MQW sample and a bulk GaAs sample at the same laser fluence generating a carrier density of $3 \times 10^{18} \mathrm{~cm}^{-3}$.

surface-space-charge field of the order of $10 \mathrm{kV} / \mathrm{cm}$ and a depletion width larger than $0.5 \mu \mathrm{m}$. The absorption length for 2-eV light is approximately $230 \mathrm{~nm}$ in bulk GaAs, so we cover $5-10$ wells within the surface field in the reflectivity experiments. The absorption length of light at $1.5 \mathrm{eV}$ is a factor of 4 larger.

The excitation with 2-eV laser pulses results in a hot electron distribution due to the large optical excess energy. We assume that the excited carrier distributions thermalize rapidly within 100 fs. These carrier distributions can be described by a Maxwell-Boltzmann distribution with a transient temperature leading to a thermal population of different electronic subbands within the wells. The exact population of each subband depends on the details of the intersubband relaxation dynamics for different well widths. ${ }^{24}$ Therefore the assumption of a thermalized distribution within the energy states of the quantum wells is rather crude. However, at excitation densities larger than a bulk density of $10^{18} \mathrm{~cm}^{-3}$ in our experiments, we assume a population of the lowest electron and hole subbands within the first 100 fs after the excitation. The energy relaxation of the hot carrier distribution in 2D may be considerably slower than in bulk GaAs. ${ }^{25}$

In contrast to the excitation with $2-\mathrm{eV}$ photons, the excitation with a Ti:sapphire laser allows us to tune the excitation energy into resonance with interband transitions. For one sample with 15-nm well width, we tune the excitation energy to the transition of the second heavy hole (hh2) to the second electron (e2) subband. Thus mainly the lowest two electron and hole subbands are populated. The results of the resonant excitation are compared to the results obtained with 2-eV excitation.

Since the barrier width of $3 \mathrm{~nm}$ is relatively large, we neglect the electronic coupling between adjacent wells, i.e., the formation of a superlattice miniband, under these experimental conditions of high excess energies and high densities. In order to verify this assumption, the same experiments are performed with samples of smaller barrier width, where electronic coupling between the wells is expected to be more pronounced.

\section{EXPERIMENTAL RESULTS AND DISCUSSION}

\section{A. Variation of the well width}

Figure 1 compares the REOS signals of the MQW with $d_{w}=15 \mathrm{~nm}$ and bulk GaAs at the same laser fluence, which 
corresponds to a maximum bulk excitation density of $3 \times 10^{18} \mathrm{~cm}^{-3}$. The data are obtained with $2-\mathrm{eV}$ excitation. Both bulk and MQW signals reveal a fast initial field change on the time scale of the pump pulse followed by a longliving oscillation. In the bulk sample, the surface field is already screened during the pump pulse at this excitation density. In the MQW sample the carrier transport is slowed down by the barriers, since the screening current requires tunneling through the barriers or thermionic emission of hot electrons over the barriers. The amplitude of the initial field screening is slightly different for the two samples, since the surface fields are not equal. The ultrafast field screening is the driving force for coherent LO phonons, which are clearly observed as periodic modulations. The amplitude of the coherent LO phonon contribution is determined by the time derivative of the polarization change during the pump pulse, which is essentially the current within the surface field. ${ }^{7,22}$ Hence the amplitude of the coherent LO phonon is larger for bulk GaAs than for the MQW. However, it is important to note that the carrier-phonon interaction discussed below only depends on the excitation density and not on the initial coherent LO phonon amplitude. ${ }^{26}$ The excitation densities within the GaAs wells of different well widths and in bulk GaAs are assumed as constant at constant laser fluence, since the absorption of 2-eV photons does not change significantly. Therefore the differences observed in the coherent phonon dynamics in bulk GaAs and MQW's of different well widths are directly related to the modification of the dimensionality of the electron gas, i.e., the change from a 2D to 3D behavior.

In Fig. 2, the phonon signals in the time domain [Fig. 2(a)] and the corresponding Fourier spectra [Fig. 2(b)] of bulk GaAs are compared to the MQW samples with different well widths. The data are recorded with the time derivative technique. In all samples coherent phonons are generated and can be traced over several picoseconds. The MQW with the smallest well $(15 \mathrm{~nm})$ reveals a sharp peak at the $\mathbf{q}=\mathbf{0}$ LO frequency of $8.75 \mathrm{THz}$. For increasing well widths, a shoulder appears at the low-energy side of the LO phonon in the vicinity of the TO phonon $(8.0 \mathrm{THz})$, while the width of the LO can be fitted by a Lorentzian with unchanged spectral width. The bulk sample reveals a broadened LO peak and an additional broad structure centered around the TO frequency. From a numerical fit to the time-domain data, we obtain a dephasing time of $4.5 \mathrm{ps} \pm 0.1 \mathrm{ps}$ for the MQW sample, which is slightly larger than the value of 4.0 ps observed for the LO mode in bulk GaAs at low excitation densities. ${ }^{5}$ LO modes from the barrier material are not observed due to the selective excitation in the wells. Effects due to confined optical phonons are not expected at the well widths and the Al content of our samples.

In bulk GaAs, the frequency spectrum is well understood. The plasma frequency of the optically excited carriers is above the LO phonon resonance. The dense carrier plasma screens the long-range Coulomb interaction of the LO phonon, thus changing its frequency to the TO frequency (screened LO mode). ${ }^{5,7}$ The bare LO mode is still observed due to probing of regions with lower carrier densities. In comparison to plasmon-phonon coupling as observed in Raman experiments, ${ }^{11}$ no coupled modes below the TO and above the LO phonon are resolved due to spatial inhomoge-
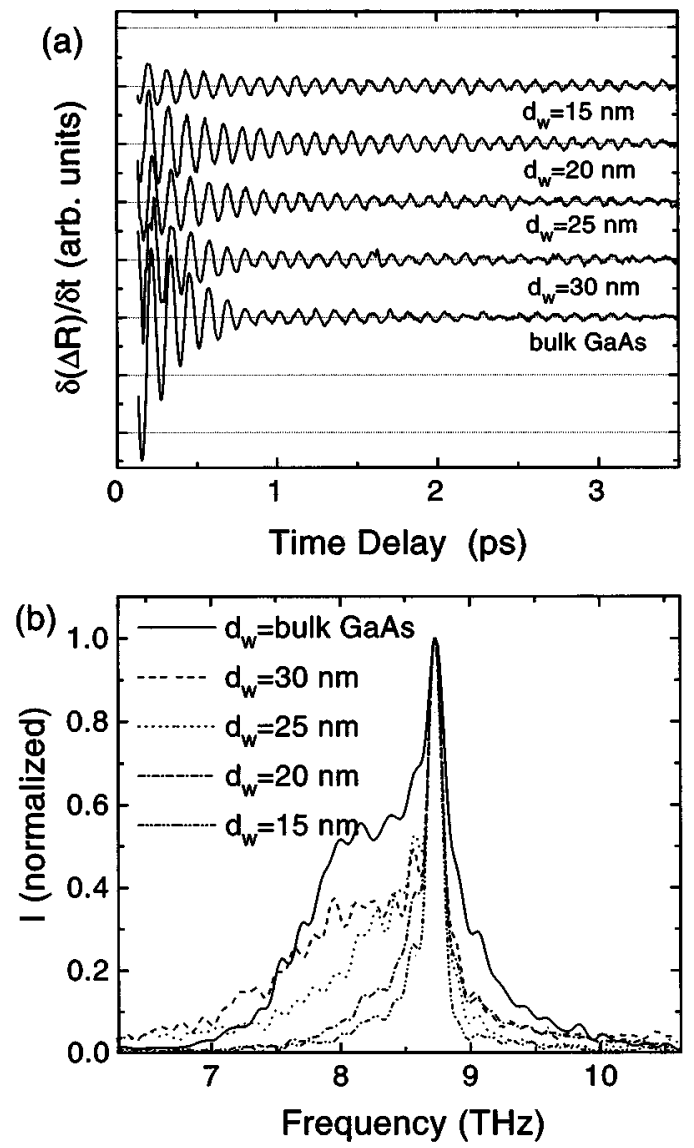

FIG. 2. (a) Coherent phonon signal and (b) Fourier transform of the bulk GaAs sample and the MQW samples with different well widths. The laser fluence is constant for all samples and corresponds to a maximum excitation density of $3 \times 10^{18} \mathrm{~cm}^{-3}$ in the bulk sample.

neities of the carrier distributions and strong damping of the nonequilibrium plasma. ${ }^{5,7}$ The dephasing time of the mode at TO frequency is in the subpicosecond range $(\approx 700 \mathrm{fs})$. Although the carrier densities are the same for the different MQW samples, the occurrence of a TO-like mode strongly depends on the well width.

In order to compare the screened LO phonon contribution to the different spectra, we subtract a Lorentzian fit to the LO peak and integrate over the remaining spectrum. This value is a measure of the intensity of the modified LO phonon resonance. In Fig. 3 the values of this integral normalized to the bulk value are shown for two different excitation densities. The higher excitation densities correspond to the data shown in Fig. $2\left(3 \times 10^{18} \mathrm{~cm}^{-3}\right)$, the lower intensity corresponds to a bulk carrier density of $2.5 \times 10^{18} \mathrm{~cm}^{-3}$. In the range of well widths investigated, the screened LO phonon contribution increases approximately linearly with the well width. The extrapolation of this linearity towards large well widths suggests that in a MQW sample with approximately $38 \mathrm{~nm}$ well width, the screened LO contribution equals the screening of the LO phonon in bulk GaAs. At the lower excitation densities the linear extrapolation to the data crosses the bulk value at a larger well width $(42 \mathrm{~nm})$. For wells smaller than $12.5 \mathrm{~nm}$ both linear fits suggest that the LO mode is not modified at all. 


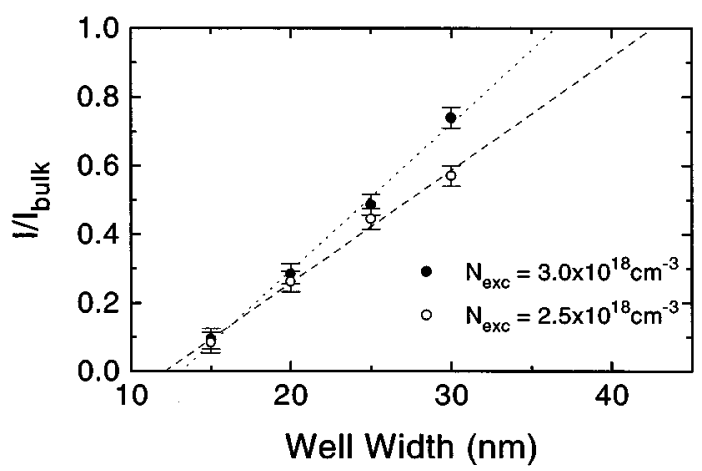

FIG. 3. Screened LO phonon contribution as a function of well width normalized to the screened LO phonon contribution in bulk GaAs for two different densities, $3 \times 10^{18} \mathrm{~cm}^{-3}$ (closed circles) and $2.5 \times 10^{18} \mathrm{~cm}^{-3}$ (open circles). The straight lines are fits to the data.

\section{B. Resonant excitation conditions}

The data shown in the preceding section are obtained with excitation at 2-eV photon energy. In order to reduce the number of populated subbands, we perform the same experiments with a fs Ti:sapphire at 1.5-eV photon energy. We show only data for the smallest well width, since the direct comparison of data obtained from samples with different well widths is more difficult than for $2-\mathrm{eV}$ excitation due to a stronger change of the excitation conditions for different samples. The comparison would require an adjustment of the photon energy to the position of the interband transitions for the different samples.

In Fig. 4 we compare extracted oscillations for the bulk GaAs sample and the quantum well with $d_{w}=15 \mathrm{~nm}$ for different excitation densities. The maximum excitation fluence results in an optically excited density of $N_{0}=3 \times 10^{18}$ $\mathrm{cm}^{-3}$ in the bulk sample. In the MQW sample, the excitation is resonant with the hh2-e 2 transition. Figure 4(a) shows the time-domain data of the REOS data of the MQW sample for three different excitation densities of $N_{0}, 0.5 N_{0}$, and $0.3 N_{0}$. For the highest excitation density a beating is clearly observed. For the lower excitation densities, the amplitude of the oscillations and the beating signature in the data decrease. The normalized Fourier transforms of the data are shown in Fig. 4(b). For the highest excitation density, clearly distinguishable peaks close to the LO phonon frequency of 8.75 $\mathrm{THz}$ and at $7.7 \mathrm{THz}$ are observed. The lower frequency is well below the TO frequency of $8.0 \mathrm{THz}$. At decreased excitation densities, the amplitude of the peak at $7.7 \mathrm{THz}$ decreases. The peak at the LO phonon frequency slightly shifts by $0.1 \mathrm{THz}$ to lower frequencies with lower excitation densities. For comparison, the normalized Fourier spectra obtained for the bulk sample are shown in Fig. 4(c). They are shown for the highest and lowest excitation intensities as used for the experiments in the MQW sample $\left(N_{0}\right.$ and $0.3 N_{0}$ ) and at a further reduced density of $0.15 N_{0}$. As in the MQW sample, two maxima close to the LO and TO frequencies can be clearly distinguished. With increasing excitation density, the maximum close to the LO frequency increases, but even for the lowest excitation density, the spectrum is still dominated by the maximum at $8.0 \mathrm{THz}$.

There are several remarkable differences between the spectra of the MQW and bulk samples at 1.5-eV excitation as well as between the comparable spectra at $2.0-\mathrm{eV}$ excitation
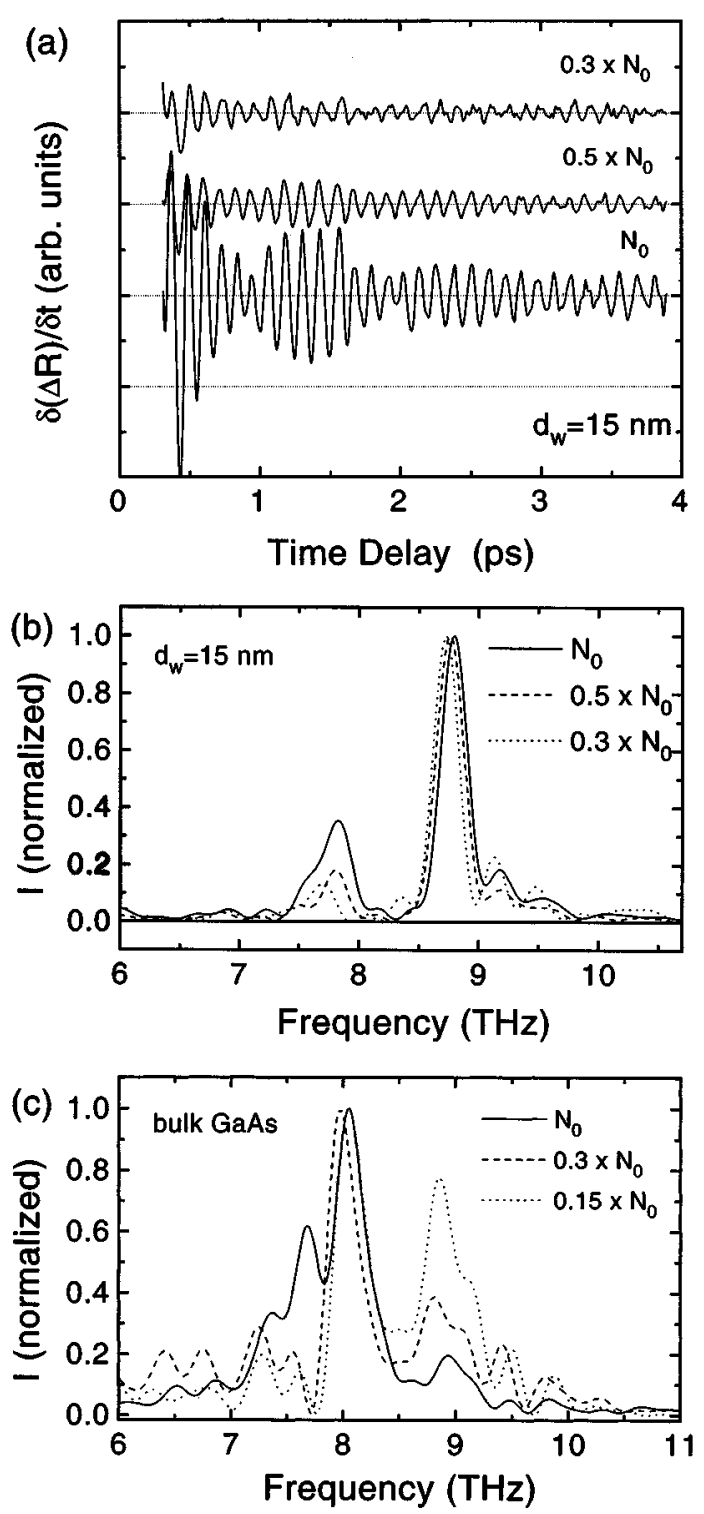

FIG. 4. (a) Time derivative REOS data of the MQW sample with $15-\mathrm{nm}$ well width at different excitation densities and $1.5-\mathrm{eV}$ photon energy. The maximum density $N_{0}$ corresponds to a bulk density of $3 \times 10^{18} \mathrm{~cm}^{-3}$. (b) Fourier transforms of the data in (a) normalized to the maximum close to the LO phonon. (c) Fourier transforms of the MQW sample normalized to the screened LO phonon contribution close to the TO frequency.

energy. First of all, in bulk as well as in the MQW sample the maxima at the LO and close to the TO frequency are clearly separated. In the bulk, this effect is based on reduced scattering rates for a "cold" plasma as compared to a "hot" plasma leading to a reduced dephasing time of the screened LO phonon contribution close to the TO frequency. At 1.5 $\mathrm{eV}$, the additional mechanism of side-valley scattering is inhibited, which is a dominant scattering mechanism for electrons excited with $2.0-\mathrm{eV}$. In addition, $2.0-\mathrm{eV}$ photons also excite holes in the split-off band of the valence band. Due to the different masses in different bands and valleys, the plasma mode is significantly broadened. This leads to the short dephasing time for the screened LO phonon contribution for $2.0-\mathrm{eV}$ excitation. For $1.5-\mathrm{eV}$ excitation the plasma 


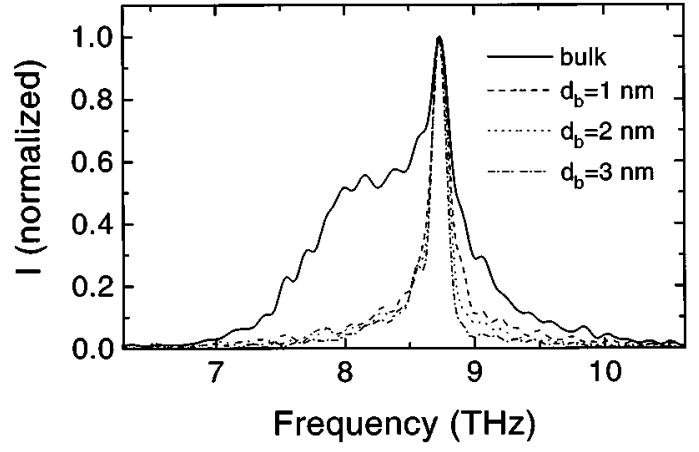

FIG. 5. Normalized Fourier transform of the coherent phonon signals of bulk GaAs and of MQW's with constant well width $d_{w}=15 \mathrm{~nm}$ and different barrier widths of $1 \mathrm{~nm}$ (dashed line), $2 \mathrm{~nm}$ (dotted line), and $3 \mathrm{~nm}$ (dashed-dotted line).

consists only of $\Gamma$-valley electrons. Therefore the scattering channels are reduced and the mass is homogeneous, leading to an effective screening of the LO phonon even at the lowest excitation densities. We plan to publish further details on the bulk plasmon-phonon coupling elsewhere. ${ }^{27}$ In the MQW sample, the observation of a clearly separated maximum at 7.7 $\mathrm{THz}$ is in strong contrast to the observation at $2.0-\mathrm{eV}$ excitation, where a broad structure between the LO and TO frequency is observed. This difference is attributed to a reduced broadening of the $2 \rightarrow 1$ intersubband transition, in contrast to the contribution of a large set of intersubband transitions with higher energies under $2.0-\mathrm{eV}$ excitation. The slight blueshift of the LO phonon resonance as well as the redshift of the low-frequency resonance below the TO frequency is explained by the theory (see Sec. V).

\section{VARIATION OF THE BARRIER WIDTH}

In order to check whether superlattice effects, i.e., electronic coupling between adjacent wells, have to be taken into account, the investigations are extended to MQW's with constant well width $(15 \mathrm{~nm})$ and smaller barrier widths $(1 \mathrm{~nm}$ and $2 \mathrm{~nm}$ ). This experiment is complementary to the previous ones, since the transition from the $2 \mathrm{D}$ to the $3 \mathrm{D}$ case can also be studied in the limit of vanishing barrier widths. The experiments are performed under excitation with laser pulses at $2 \mathrm{eV}$. The Fourier spectra for this sample are compared to the bulk and the $d_{b}=3 \mathrm{~nm}$ sample as shown in Fig. 5. The spectra show no enhanced TO contribution with decreasing barrier width, in accordance with the assumption that coupling between adjacent wells can be neglected under these specific excitation conditions. This observation confirms that the LO phonon resonance is modified by the electronic susceptibility of the wells as defined by their width. However, the LO peak broadens slightly with decreasing barrier width. This broadening is related to a decrease in the LO phonon dephasing time from 4.5 -ps for $d_{b}=3 \mathrm{~nm}$ to $4 \mathrm{ps}$ for $d_{b}=1$ $\mathrm{nm}$. The dephasing time of $4 \mathrm{ps}$ is in accordance with the dephasing time found from time-resolved Raman experiments $^{28,29}$ as well as from coherent phonon investigations ${ }^{5}$ in bulk GaAs at low carrier densities. Therefore the $4.5 \mathrm{ps}$ dephasing time in the MQW samples is larger than the largest dephasing times observed in bulk GaAs. In bulk, the dephasing of the coherent amplitude is mainly given by the lifetime of the phonons defined by anharmonic decay of optical phonons into acoustic phonons. This fact suggests that the anharmonic decay channels are altered in the MQW structure. Further investigations are in progress to reveal deeper insight into the mechanism, which accounts for the decrease of the LO phonon dephasing time at smaller barrier widths.

\section{THEORETICAL CONSIDERATIONS}

The experimentally observed oscillatory signals indicate that we coherently excite some of the longitudinal eigenmodes of the system. To determine the nature and the properties of the eigenmodes, we need to evaluate the dielectric properties of our samples.

We are only interested in the dielectric properties in the growth direction. The contribution of carriers to the dielectric function can be expressed in terms of the subband energies $\varepsilon_{\alpha i}$ and the dipole matrix elements between the subbands $d_{i j}^{\alpha}$, where $i$ and $j$ are the subband indices, and $\alpha$ is the band index (we will consider the conduction band and the heavy-hole band, $\alpha=c, h):{ }^{12}$

$$
\epsilon(\omega)=\epsilon_{\infty}+4 \pi \sum_{\alpha, i, j} \frac{\left|d_{i j}^{\alpha}\right|^{2}\left(n_{i}^{\alpha}-n_{j}^{\alpha}\right)}{\varepsilon_{i}^{\alpha}-\varepsilon_{j}^{\alpha}+\omega},
$$

where we have added the background dielectric constant $\epsilon_{\infty}$. The densities $n_{i, j}^{\alpha}$ represent the total (summed over perpendicular momenta) number of electrons in a given subband. To include the effect of the LO phonons on the dielectric properties, $\epsilon_{\infty}$ needs to be replaced by the dielectric function of the phonons: ${ }^{19}$

$$
\epsilon_{\mathrm{ph}}(\omega)=\epsilon_{\infty}\left[\omega_{\mathrm{LO}}^{2}-\omega^{2}\right] /\left[\omega_{\mathrm{TO}}^{2}-\omega^{2}\right] .
$$

In our optical excitation experiments, the laser pulse creates a highly nonequilibrium initial distribution that rapidly thermalizes and later cools to the lattice temperature on a picosecond time scale. To avoid solving a complicated problem of the energy relaxation of carriers, in this work we will assume thermal distributions for electrons and holes while allowing the electron and hole temperatures to be different. The initial temperature of the carrier distributions is determined by the optical excess energy, which is about $80 \mathrm{meV}$ for $1.5-\mathrm{eV}$ excitation and $580 \mathrm{meV}$ for excitation with $2-\mathrm{eV}$ photons. In general, the electron temperature can be much higher than the hole temperature due to the lower confinement energies of the holes and their higher effective masses (for the heavy holes). In the calculations below, we add phenomenological dephasing terms $i \gamma_{\mathrm{el}}(2 \mathrm{meV})$ and $i \gamma_{\mathrm{ph}}(0.1$ $\mathrm{meV}$ ) to the frequencies in (2) and (3) to account for relaxation processes.

Figure 6 shows the electronic component of the dielectric function (2) evaluated for a relatively narrow well of $15 \mathrm{~nm}$. The peaks in the imaginary parts correspond to different transition energies in (2). A closely spaced group of peaks below $10 \mathrm{THz}$ corresponds to the intersubband transitions in the heavy-hole band, while the peaks at 18 and $31 \mathrm{THz}$ correspond to $1 \rightarrow 2$ and $2 \rightarrow 3$ transitions between the conduction subbands. Note that generally only $i \rightarrow i+1$ transitions give noticeable contributions to (2) since the intersubband dipole matrix elements $d_{i j}$ are largest for transitions between 


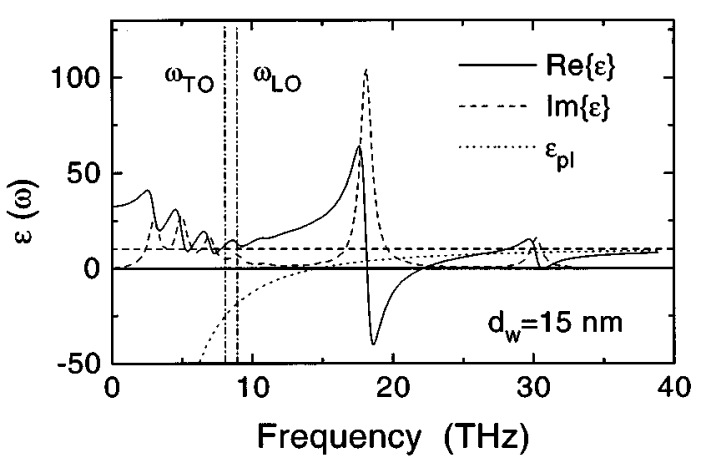

FIG. 6. Electronic component of the dielectric function obtained from Eq. (2) for a narrow 15-nm well $\left(N=2 \times 10^{18} \mathrm{~cm}^{-3}\right.$, electron and hole temperatures are $300 \mathrm{~K}$ ). Real part of $\epsilon$ (solid line), imaginary part (dashed line), and the real part of the contribution to the dielectric function of a plasma $\epsilon_{\mathrm{pl}}$ at the same density (dashed line). The two vertical lines indicate the TO and LO frequency, respectively, and the dashed horizontal line $\epsilon_{\infty}$.

neighboring subbands and drop off rapidly as a function of $|i-j|$. If an intersubband transition is sufficiently isolated from others like the one at $19 \mathrm{THz}$, the dielectric function can cross zero in its vicinity (at $22 \mathrm{THz}$ ), giving rise to a new collective longitudinal mode (intersubband plasmon). ${ }^{10,18-20}$

As we will see below, the observed signal in our experiments is largely determined by the electronic contribution to the dielectric function in the spectral region around the phonon frequency. For narrow wells, one can see from Fig. 6 that this contribution tends to be small because the phonon frequency falls in between the low-frequency transitions caused by the holes and the higher-frequency conduction band transitions. As a result, the holes give a negative contribution to the dielectric function at the phonon frequency while the electrons give a positive contribution which to a large extent compensate each other.

When the well width is increased, the frequencies of intersubband transitions decrease as $1 / d_{w}^{2}$, and some of the electronic intersubband transitions move below the phonon frequency (Fig. 7). The first such crossing occurs at approximately $230 \AA$. As is seen in Fig. 7, when a transition moves below the phonon energy, its contribution at the phonon frequency becomes negative and adds constructively with the

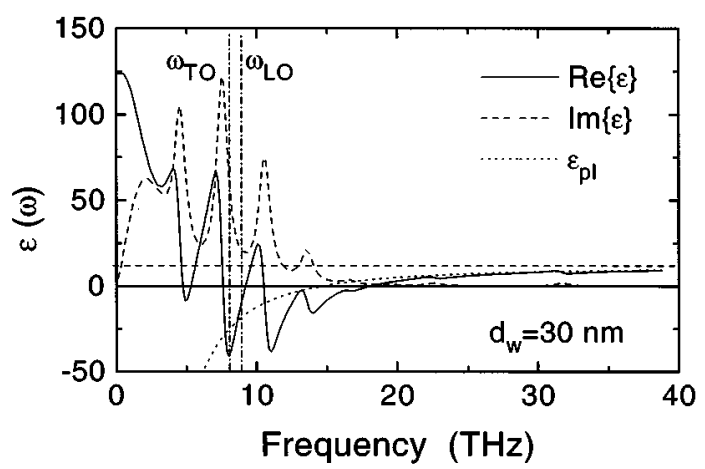

FIG. 7. Electronic component of the dielectric function obtained from Eq. (2) for a wide 30-nm well $\left(N=2 \times 10^{18} \mathrm{~cm}^{-3}\right.$, electron and hole temperatures are $300 \mathrm{~K}$ ). The curve types are the same as in Fig. 6. contribution of the hole band. In the bulk, the contribution of the electrons to the dielectric function is given by the plasmon dispersion:

$$
\epsilon_{\mathrm{pl}}(\omega)=\epsilon_{\infty}-\omega_{\mathrm{pl}}^{2} / \omega^{2},
$$

where $\omega_{\mathrm{pl}}^{2}=4 \pi N e^{2} / m^{*}, m^{*}$ is the reduced mass, and $N$ is the total density. This contribution to the dielectric function is shown by the dashed line in Figs. 6 and 7 and is always negative. Therefore the overall effect of electronic screening in a wide well and in the bulk is qualitatively similar. Note that at frequencies above the highest intersubband transition the dielectric function of the well [Eq. (2)] approaches the bulk plasmon dispersion (Fig. 7). This is a general result that follows from the $f$-sum rule. Consequently, as the well width increases and the intersubband frequencies tend to zero, the dielectric function (2) continuously evolves toward the bulk plasmon dispersion (4).

The dielectric function contains information about the transient response of the system to sudden changes in the external conditions. Before the ultrafast optical excitation, the superlattice is subjected to the built-in dc field $D$ and has a dc polarization given by $P=(D-E) / 4 \pi$ $=D\left[1-1 / \epsilon_{0}(\omega=0)\right] / 4 \pi$, where $\epsilon_{0}$ is the dielectric function of the system before the excitation, i.e., for zero density of electrons. The excitation pulse very rapidly creates a finite density of carriers in the sample and thus modifies its dielectric function. As a result, the old value of the polarization can no longer be supported by the system and will relax to a new value corresponding to the new dielectric function. The same transient can be achieved if the system always had the "new" dielectric function $\epsilon(\omega)$ [Eq. (2)] but was subjected to a higher applied field $D^{\prime}=D \epsilon(\omega=0) / \epsilon_{0}$ prior to excitation. Assuming that the change is instantaneous [which is a reasonable approximation as long as the excitation duration (50 fs) is much shorter than the period of the relevant oscillations (113 fs)], we can immediately write down the Fourier transform of the transient electric field component caused by the excitation:

$$
E_{\mathrm{tr}}(\omega)=\frac{D}{i \omega}\left[\frac{\epsilon(0)-\epsilon_{0}}{\epsilon_{0}}\right]\left(\frac{1}{\epsilon(\omega)}-\frac{1}{\epsilon(0)}\right) .
$$

According to (1), this quantity is directly related to the observed REOS signal. As was recently demonstrated, ${ }^{7}$ for the case of LO phonons one has to go beyond Eq. (1) and consider a direct contribution of the lattice displacement $W$ of the LO mode to the observed signal. The lattice displacement is related to the field,

$$
W(\omega)=\frac{\gamma_{12}}{\left(\omega_{\mathrm{TO}}^{2}-\omega^{2}\right)} E(\omega),
$$

and the observed response is proportional to a linear combination of (5) and (6). Since $\epsilon(\omega)$ is known [we evaluate it by adding electronic contribution (2) and the phonon contribution (3)], we can predict the observed signals in the frequency domain.

In Fig. 8 we plot the normalized reflective response evaluated by the above procedure for different well widths. The results are consistent with experimental observations of Fig. 2 - we predict that for narrow wells the LO phonon is 


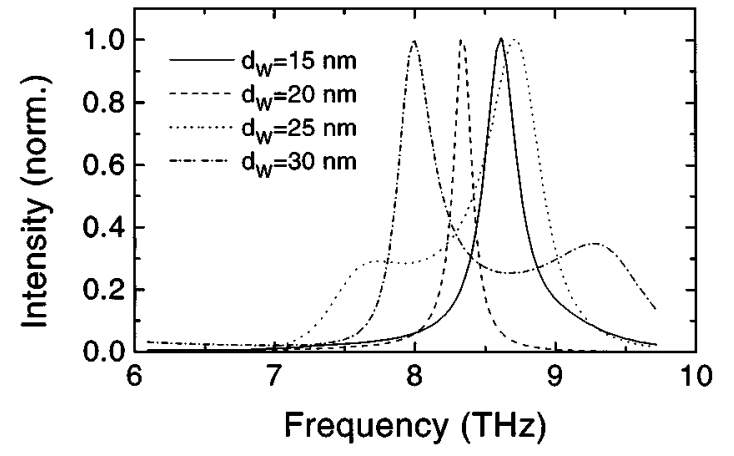

FIG. 8. Normalized frequency components of REOS signals calculated for different well widths at a density of $N=2 \times 10^{18}$ $\mathrm{cm}^{-3}$, electron and hole temperatures are $300 \mathrm{~K}$.

practically unscreened, and that for wide wells there is a strong signal at around the TO frequency $(8 \mathrm{THz})$. As in the bulk case, ${ }^{7}$ the direct comparison of theory and experiment is complicated by the fact that the low-density wings of the excited spot give a strong contribution to the response in the form of a bare LO phonon peak, which should be superimposed on the calculated response shown in Fig. 8. For a $15-\mathrm{nm}$ well the phonon line is practically unchanged because the electronic contribution (2) is small at the phonon frequency (see Fig. 6). For a 20-nm well the theory predicts that the phonon line should be shifted down in frequency because for this well width the phonon frequency is right below the $1 \rightarrow 2$ conduction band transition which gives a strong positive contribution at the phonon frequency and shifts the zero of the dielectric function down from $\omega_{\mathrm{LO}}$ into the forbidden gap between $\omega_{\mathrm{LO}}$ and $\omega_{\mathrm{TO}}$. This downward shift should deform the bare phonon line and create a low-frequency shoulder that is close to the experimental observations of Fig. 2. For wider wells $(25$ and $30 \mathrm{~nm}$ ) some of the conduction band transitions are already below the phonon frequency and thus the contribution to the dielectric function is negative. A negative contribution has an effect similar to that of a bulk plasmon - it shifts the LO resonance up in frequency and can also create an additional resonance below $\omega_{\text {TO }}$ which is seen in Fig. 8. A strong broadband signal around the TO frequency is also present in the experiment (Fig. 2) for the 30-nm well. Our model also accounts for the density dependence of the transition from the 2D screening to 3D plasmon-phonon coupling as deduced from the linear fits in Fig. 3, where for a higher density the bulklike behavior is expected to be obeserved at a smaller well width.

The experimental data for resonant excitation (Fig. 4) suggest that the electronic contribution at the phonon frequency is negative (blueshift of the LO phonon line and a new resonance below $\omega_{\mathrm{TO}}$ ). To achieve a negative contribution, we have to assume that the distributions of electrons and holes are nonthermal: for resonant excitation the initial populations of the first and the second conduction subbands are practically equal, which strongly suppresses the $1 \rightarrow 2$ transition and reduces its positive contribution (see Fig. 6). The only way we can incorporate that into our model at the present time is to set the electron temperature to a very high value $(1000 \mathrm{~K})$ to equalize the population of the lower subbands. We also set the hole temperature below the room tem-

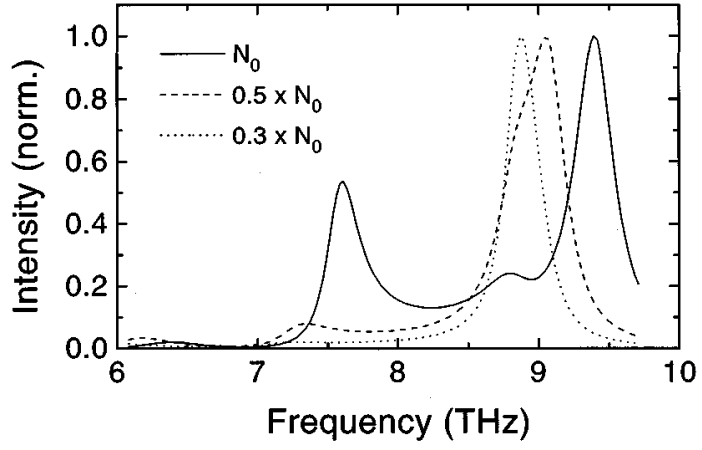

FIG. 9. Normalized frequency components of REOS signals calculated for different densities in a narrow well $(15 \mathrm{~nm})$. The populations of the electron and hole subbands are adjusted via an electron temperature of $1000 \mathrm{~K}$ and a hole temperature of $150 \mathrm{~K}$.

perature because the holes are created with excess energy of about $10 \mathrm{meV}$; this suppresses the higher-lying $3 \rightarrow 4$ and $4 \rightarrow 5$ transitions in the heavy-hole band that are almost resonant with the phonon in Fig. 6. The resulting density dependence of the calculated REOS signal is shown in Fig. 9 and is qualitatively similar to the experimental observations. For this calculation, we have also reduced the phenomenological dephasing by a factor of 2 to reflect the fact that fewer relaxation channels are available for low-energy electrons.

It would be very difficult to achieve a better quantitative agreement between theory and experiment here because this would require much more effort to model realistic switching dynamics, spatial distribution of the density, ${ }^{7}$ and the detailed description of carrier relaxation. However, the general qualitative picture of the transition between very weak screening for narrow wells to the strong bulklike screening in the wide wells is largely insensitive to the details of the calculation. Therefore we believe that our model accounts for the observations reasonably well.

\section{CONCLUSION}

We reported on the observation of coherent LO phonons and their coupling to collective carrier excitations in GaAs quantum wells oscillating perpendicular to the wells. The strength of the coupling strongly depends on the well width due to the modification of the LO phonon resonance by the electronic susceptibility of the wells due to the population of several subbands. The coupling is suppressed for small well widths, since the energy spacings of the resonances are larger than the LO phonon energy. At larger well widths, the intersubband transitions come into resonance with the LO phonon, leading to new eigenfrequencies. The dephasing time of the new resonances is in the subpicosecond range due to the damping of the intersubband transitions. Under excitation of the whole set of subbands with high excess energy the contribution of several subbands leads to a broad contribution in the coherent amplitude between the LO and TO frequencies. Under resonant excitation of the two lowest subbands a sharp feature close to the TO frequency is observed. We presented a theory that is based on a model for the dielectric response perpendicular to the layers of the MQW's. Under excitation with an ultrashort laser pulse, coupled intersubband 
plasmon-phonon resonances are considered to strongly modify the dielectric response of the MQW. The main experimental observations are in qualitative agreement with the theory. Differences between the experimental results and the theory are attributed to a thermal population of the different subbands assumed in the theory. The presented method opens a way for the subpicosecond time-resolved study of carrier-phonon interaction in semiconductor heterostructures.

*Present address: Department of Physics, Ohio State University, Columbus, OH 43210.

${ }^{1}$ See e.g., contributions to Light Scattering in Semiconductor Structures and Superlattices, Vol. 273 of NATO Advanced Study Institute, Series B: Physics, edited by D.J. Lockwood and J.F. Young (Plenum, New York, 1991).

${ }^{2}$ J. Shah, in Spectroscopy of Nonequilibrium Electrons and Phonons, edited by C.V. Shank and B.P. Zakharchenya (Elsevier, Amsterdam, 1992), pp. 57-112.

${ }^{3}$ G.C. Cho, W. Kütt, and H. Kurz, Phys. Rev. Lett. 65, 764 (1990).

${ }^{4}$ T.K. Cheng, S.D. Brorson, A.S. Kazeroonian, J.S. Moodera, G. Dresselhaus, M.S. Dresselhaus, and E. Ippen, Appl. Phys. Lett. 57, 1004 (1990).

${ }^{5}$ W.A. Kütt, W. Albrecht, and H. Kurz, IEEE J. Quantum Electron. QE28, 2434 (1992).

${ }^{6}$ G.O. Smith, T. Juhasz, W.E. Bron, and Y.B. Levinson, Phys. Rev. Lett. 68, 2366 (1992).

${ }^{7}$ A.V. Kuznetsov and C.J. Stanton, Phys. Rev. B 51, 7555 (1995).

${ }^{8}$ W. Sha, A.L. Smirl, and W.F. Tseng, Phys. Rev. Lett. 74, 4273 (1995).

${ }^{9}$ Recently, coherent acoustic phonons have been observed in GaAs/AlAs superlattices: A. Yamamoto, T. Mishina, Y. Masumoto, and M. Nakayama, Phys. Rev. Lett. 73, 740 (1994).

${ }^{10}$ E. Burstein, A. Pinczuk, and D.L. Mills, Surf. Sci. 98, 451 (1980).

${ }^{11}$ A. Mooradian and G.B. Wright, Phys. Rev. Lett. 16, 999 (1966).

${ }^{12}$ S. Das Sarma and J.J. Quinn, Phys. Rev. B 25, 7603 (1982).

${ }^{13}$ D. Olego, A. Pinczuk, A.G. Gossard, and W. Wiegmann, Phys. Rev. B 25, 7867 (1982).

\section{ACKNOWLEDGMENTS}

We thank H.J. Bakker for fruitful discussions. This work was supported by the Deutsche Forschungsgemeinschaft (Grant No. Ku540/15-1) and the Volkswagen Stiftung. A.K. acknowledges support from ONR through Grant No. N00091-JJ-1956, and from NSF through Grant No. DMR 8957382.

${ }^{14}$ A.V. Chaplik, Surf. Sci. Rep. 5, 289 (1985).

${ }^{15}$ D. Heitmann, Surf. Sci. 170, 332 (1986).

${ }^{16} \mathrm{~T}$. Egeler, in Festkörperprobleme/Advances in Solid State Physics 31, edited by U. Rössler (Vieweg, Braunschweig, 1991), p. 315.

${ }^{17}$ P. Brockmann, J.F. Young, P. Hawrylak, and H.M. van Driel, Phys. Rev. B 48, 11423 (1993).

${ }^{18}$ W.L. Bloss, Solid State Commun. 46, 143 (1983).

${ }^{19}$ A.C. Tselis and J.J. Quinn, Phys. Rev. B 29, 3318 (1984).

${ }^{20}$ R. Sooryakumar, A. Pinczuk, A. Gossard, and W. Wiegmann, Phys. Rev. B 31, 2578 (1985).

${ }^{21}$ J.F. Ryan and M. Tatham, Solid State Electron. 32, 1429 (1989).

${ }^{22}$ L. Min and R.J.D. Miller, Appl. Phys. Lett. 56, 524 (1990); T. Dekorsy, T. Pfeifer, W. Kütt, and H. Kurz, Phys. Rev. B 47, 3842 (1993).

${ }^{23}$ T. Pfeifer, T. Dekorsy, W. Kütt, and H. Kurz, Appl. Phys. A 55, 482 (1992).

${ }^{24}$ S. Hunsche, K. Leo, H. Kurz, and K. Köhler, Phys. Rev. B 50, 5791 (1994), and references therein.

${ }^{25}$ Y. Rosenwaks, M.C. Hanna, D.H. Levi, D.M. Szmyd, R.K. Ahrenkiel, and A.J. Nozik, Phys. Rev. B 48, 14675 (1993).

${ }^{26}$ This has been verified in a study of the coherent phonon dephasing in bulk GaAs samples with different built-in fields.

${ }^{27}$ G.C. Cho, T. Dekorsy, T. Pfeifer, and H. Kurz (unpublished).

${ }^{28}$ J.A. Kash, S.S. Jha, and J.C. Tsang, Phys. Rev. Lett. 58, 1869 (1987).

${ }^{29}$ F. Valee, Phys. Rev. B 49, 2460 (1994). 